\title{
Risk as a primitive: a survey of measures of perceived risk
}

\author{
H.W. Brachinger ${ }^{1}$, M. Weber ${ }^{2}$ \\ 1 Seminar für Statistik/Séminaire de Statistique, University of Fribourg, Avenue de Beauregard 13, CH-1700 Fribourg, Switzerland \\ 2 Lehrstuhl für Allgemeine BWL, Finanzwirtschaft, insbesondere Bankbetriebslehre, University of Mannheim, D-68131 Mannheim, Germany
}

Received: 9 July 1996 / Accepted: 30 January 1997

\begin{abstract}
The concept of risk is essential to many problems in economics and business. Usually, risk is treated in the traditional expected utility framework where it is defined only indirectly through the shape of the utility function. The purpose of utility functions, however, is to model preferences. In this paper, we review those approaches which directly model risk judgements. After a short review of naive risk measures used in earlier economic literature, we present recent theoretical and empirical developments.

Zusammenfassung. Risiko ist ein Konzept, das bei der Behandlung vieler volks- oder betriebswirtschaftlicher Probleme eine wesentliche Rolle spielt. Üblicherweise wird Risiko im Rahmen des traditionellen Erwartungsnutzenmodells behandelt, bei dem es nur indirekt über die Form der Nutzenfunktion erfaßt wird. Der Zweck von Nutzenfunktionen besteht aber darin, Präferenzen zu modellieren. In diesem Aufsatz wird ein Überblick über solche Ansätze gegeben, die Risikowahrnehmungen direkt modellieren. Nach einer kurzen Darstellung naiver Risikomaße, die aus der früheren ökonomischen Literatur bekannt sind, werden neuere theoretische und empirische Konzepte präsentiert.
\end{abstract}

Key words: Risk judgement, perceived risk, axiomatic measures of risk

Schlüsselwörter: Risikobeurteilung, wahrgenommenes Risiko, axiomatische Risikomaße

\section{Introduction}

The term risk obviously plays a pervasive role in much of the current writings on economic, political, social, and technological issues. In all of these fields, risk is a kind of negative feature characterizing a decision alternative. Risk is meant to be a chance of injury or loss connected with a given action. However, risk is not an objective feature of a decision alternative. It is an inherently subjective construct because

Correspondence to: H.W. Brachinger what is considered a loss and what its significance and its chance of occurring is, is peculiar to the person concerned.

In the economic as well as in the psychological literature on decision making, there are various attempts to define or to characterize risk for purpose of descriptive as well as of prescriptive theory. There is a growing interest in what constitutes the risk of an alternative and how to measure it. Thereby, the main emphasis lies on the risk itself of an alternative, independently of the problem of risk preference. Risk refers to the riskiness of an alternative. It is a matter of perception or estimation. Risk preference refers to the preferability of an alternative under conditions of risk and is a matter of preferences. In such situations, risk is only one significant aspect of the available options. The decision maker's preference for a certain action, generally, also depends on other positive or perhaps additional negative features.

In this paper, we start from the assumption that there exists a meaningful risk ordering which can be obtained directly by asking an individual to judge which of a given pair of comparable alternatives is riskier. The key concept will therefore be a binary relation $\succsim$, with $A \succsim B$ meaning that an alternative $A$ is at least as risky as another alternative $B$. In general, this relation is assumed to be a weak order, i.e. strictly complete and transitive. Only in Sect. 11 completeness is resigned. Throughout the paper the relation $A \succ B$ states that alternative $A$ is riskier than alternative $B$ while $A \sim B$ means that $A$ and $B$ are equally risky. Thereby, $\sim$ and $\succ$ denote the symmetric and the asymmetric parts of $\succsim$, respectively. The risk ordering - derived from judgements about perceived risk - need not be related to the individual's preference ordering in any simple way.

According to the conception of standard measurement theory, we are looking for functions $R$ which numerically represent the risk ordering $\succsim$, i.e. functions $R$ with the property

$A \succsim B \quad \Longleftrightarrow \quad R(A) \geq R(B)$.

Every such function $R$ will be called risk measurement function or simply risk measure. Note that we take a subject's risk judgement as a primitive. As we are interested in measures of perceived risk, we cannot and we should not give 
an abstract definition of risk. It is peoples' perception which ultimately determines the definition of risk.

There are three main reasons which necessitate a means for the direct comparison of alternatives as to their risk. First, the understanding of riskiness judgements might help to understand preference. Taking risk and value as primitives, one might explain preference by a risk-value model, i.e. by a function of these two components. Many theories in management and finance rely on such a separate consideration of risk and value. Possibly the best known example is modern portfolio selection theory as developed by Markowitz [48] and others. Within this context, the decision problem is viewed as choosing among possible risk-return combinations and formulated as either maximizing return for a given level of risk or minimizing risk for a given level of return. With such an approach, obviously, the decision will generally depend on the risk measure used. Second, there is growing empirical evidence that, under conditions of uncertainty, people base their decisions on qualitative aspects of choice alternatives such as risk. For a study from the practice of investment decision making see, e.g., Brachinger and Schubert [15]. Finally, judgements of perceived risk may be required as such, independent of the necessity of choice, e.g., for intervention before the decision stage in a public policy setting. People talk all the time about the riskiness of things like nuclear energy or how risky it might be not taking an afternoon nap.

Despite the importance of risk, there is little consensus on its definition. In empirical studies, typically, two dimensions which appear to determine perceived risk have been identified: amount of potential loss and probability of occurrence of loss. The risk of an alternative increases if the probability of loss increases or if the amount of potential loss increases. Unfortunately, up to now no agreement has been reached on the relative importance of the uncertainty of outcomes versus their undesirability for determining perceived risk. Furthermore, there is empirical evidence that possible gains reduce the perceived risk of an alternative. But it is by no means clear how and to what extent risk perception depends on potential gains. Other empirical studies have shown that risk is not simply equal to something like negative preference, it is an own important concept. E.U. Weber, Anderson and Birnbaum [67], e.g., suggest that people, when judging the riskiness of an alternative, encode and combine probability and outcome information in qualitatively different ways than when judging its attractiveness.

Having accepted that risk is something different from risk preference, it would be interesting to know what the relation between risk and risk preference is. There are various theories of decision making under risk. Some of these theories like risk-value models make explicit use of a risk measure, others do not. Within the framework of the Expected Utility Model, e.g., a single alternative's risk is not quantified, only an individual's general attitude towards risk is reflected by the shape of his or her utility function and, given the utility function, quantified by the well-known ArrowPratt measure. Nevertheless, we will neither review the literature on risk-value models nor discuss the relation of risk measures to utility based theories of decision making under risk. For an overview on risk-value models and their relation to utility based theories of decision making under risk see Sarin and Weber [54]. We want to concentrate on one important component of risk-value models, the factor risk, independently of any risk preferences. The literature on risk and its measurement is relatively new, scattered in different fields and covering theoretical as well as empirical work.

We also do not want to review the vast amount of literature dealing with the psychology of risk judgements. See Slovic [58] and Bayerische Rück [6] for an overview or Burgemeister and Weber [16] for an application. It is definitely interesting to know that people in Hungary are more afraid of collecting and eating mushrooms than US-citizens and even more important how these judgements can be explained. In our overview, we want to concentrate on how people evaluate the riskiness of lotteries, the fruit flies of modern economics. Risk measurement as it will be presented in our paper seeks to get behind specific contextual referents of risky alternatives to consider characteristics of risk that apply to many different situations.

It is the objective of our paper, first, to review the more naive risk measures which have been used in the earlier economic literature and for which no strict theoretical foundations have been given. Then an overview is given on recently developed economic or psychological theories of perceived risk which rely on the axiomatic approach of modern measurement theory. (see also Brachinger [10], Weber [68], as well as E.U. Weber and Bottom [65]). In addition to a theoretical discussion of risk measurement, we will review the empirical work investigating jugdements of perceived riskiness.

\section{Naive risk measures}

In the economic, especially the finance literature, traditionally, the risk of an option has primarily been associated with the dispersion of the corresponding random variable. Therefore, not later than since Markowitz's [47, 48] and Tobin's [61] pioneering work on portfolio selection, it is common to measure the riskiness of an alternative by the variance $\sigma^{2}$ or the standard deviation $\sigma$ of its outcomes. Let an alternative's future wealth be characterized by a random variable $\tilde{x}$ with distribution function $F_{\bar{x}}$ and probability density function $f_{\tilde{x}}$. Then, with the mathematical expectation

$\mu:=E(\tilde{x}):=\int_{-\infty}^{+\infty} x d F_{\tilde{x}}(x)$

these risk measures are defined by

$\sigma^{2}:=\operatorname{var}(\tilde{x}):=\int_{-\infty}^{+\infty}(x-\mu)^{2} d F_{\tilde{x}}(x)$

and

$\sigma:=\left[\int_{-\infty}^{+\infty}(x-\mu)^{2} d F_{\tilde{x}}(x)\right]^{1 / 2}$.

In addition, sometimes similar naive risk measures are discussed (cf. Markowitz [48, pp. 286-297]). Within these are the expected absolute deviation around $\mu$ 


$$
\int_{-\infty}^{+\infty}|x-\mu| d F_{\tilde{x}}(x)
$$

and the expected absolut deviation around 0

$$
\int_{-\infty}^{+\infty}|x| d F_{\tilde{x}}(x)
$$

Besides, it has been conventional wisdom in economics and other fields of research that risk is the chance of something bad happening. In this vein, risk is associated with an outcome that is worse than some specific target outcome and its probability. Within the risk measures tailored to this notion of risk are the lower semivariance

$$
\int_{-\infty}^{\mu}(x-\mu)^{2} d F_{\tilde{x}}(x)
$$

the expected value of loss

$$
-\int_{-\infty}^{0} x d F_{\tilde{x}}(x)
$$

and the probability of loss or probability of ruin

$P_{\tilde{x}}(\tilde{x} \leq r)=\int_{-\infty}^{r} d F_{\tilde{x}}(x)$

Thereby, $r$ is a certain target level outcomes lower of which are a loss or disastrous to the decision maker.

Stone $[59,60]$ has shown that all of these risk measures are special cases of one of two related three-parameter families of risk measures. The first three-parameter risk measure is defined as

$R_{S 1}(\tilde{x}):=\int_{-\infty}^{q\left(F_{\tilde{x}}\right)}\left|x-p\left(F_{\tilde{x}}\right)\right|^{k} d F_{\tilde{x}}(x) \quad(k \geq 0)$,

where $p\left(F_{\tilde{x}}\right)$ denotes a reference level of wealth from which deviations are measured. The positive number $k$ specifies a power to which deviations in wealth from the reference level are raised and thus $k$ is a measure of the relative impact of large and small deviations. The parameter $q\left(F_{\tilde{x}}\right)$ is a range parameter that specifies what deviations are to be included in the risk measure. The second three-parameter risk measure is defined to be the $k^{t h}$ root of $R_{S 1}(\tilde{x})$, i.e.,

$R_{S 2}(\tilde{x}):=\left[\int_{-\infty}^{q\left(F_{\tilde{x}}\right)}\left|x-p\left(F_{\tilde{x}}\right)\right|^{k} d F_{\tilde{x}}(x)\right]^{1 / k} \quad(k>0)$.

Through appropriate choices of the parameters $p\left(F_{\tilde{x}}\right)$, $q\left(F_{\tilde{x}}\right)$, and $k$ it is easy to see that the above listed risk measures are special cases of one of Stone's families. Equation (10), e.g., gives the semivariance when $k=2$ and $p\left(F_{\tilde{x}}\right)=q\left(F_{\tilde{x}}\right)=\mu$. A further interesting special case of Stone's family (10) of risk measures is the generalized risk measure
$R_{F 1}(\tilde{x})=\int_{-\infty}^{t}(t-x)^{\alpha} d F_{\tilde{x}}(x) \quad(\alpha>0)$

proposed by Fishburn [28] where $t$ is a fixed upper target. This measure results from $(10)$ if one chooses $p\left(F_{\tilde{x}}\right)=$ $q\left(F_{\tilde{x}}\right)=t$. The parameter $\alpha$ of Fishburn's risk measure $R_{F 1}$ may as well as the parameter $k$ in Stone's families be interpreted as risk-parameter characterizing the decision-maker's risk attitude. Values $\alpha>1$ describe a kind of risk-sensitive, values $\alpha \in(0,1)$ a kind of risk-insensitive behavior (see also Albrecht [1]).

Other naive risk measures scattered in the literature are the Shannon entropy

$$
-\int_{-\infty}^{+\infty} f_{\tilde{x}}(x) \ln \left(f_{\tilde{x}}(x)\right) d x
$$

which is well-known from communication theory (cf. Machina and Rothschild [46, p. 203]), the interquartile range $F_{\tilde{x}}^{-1}(0.75)-F_{\tilde{x}}^{-1}(0.25)$, and the minimum outcome $-x_{\min }$ of $\tilde{x}$ (cf. Schneeweiss [55, p. 60]). For cases where values $x<0$, i.e. losses are possible, the minimum outcome is usually called maximum loss (cf. Markowitz [48, p. 287]).

\section{Coombs' psychophysics of risk}

Among the first to approach the problem of risk itself, independently of the problem of risk preference, were Coombs and Huang [23]. They discuss two-outcome gambles of the form $g=(y, p, z)$, where two amounts $y$ and $z$ of money, $y \geq z$, can be won with probability $p$ and $q=1-p$, respectively. Let $\Omega_{p}$ denote the space of all such two-outcome gambles with fixed winning probability $p$. Then, Coombs and Huang consider the transformed gambles

$a(g):=(y+a, p, z-a p / q), \quad a \in A$

$b(g):=(y+b, p, z+b), \quad b \in B$

and

$c(g):=(y, p, z)^{(c)}, \quad c \in C$,

where $A, B$ are sets of real numbers and $C$ is a set of natural numbers. $(y, p, z)^{(c)}$ designates that the gamble $g$ is played $c$ times independently. It is easy to show that the transformation $a(\cdot)$ is expectation-preserving, and that a gamble's variance increases with $a$. Defining a gamble's $g$ expected regret by $(1-p)(y-z)$, the amount $a$ specifies the increase in expected regret caused by the transformation $a(\cdot)$. The transformation $b(\cdot)$ is variance-preserving, but increases a gamble's expectation by the amount $b$. Furthermore, multiple play leads to multiplying expectation correspondingly.

Coombs and Huang show that any gamble $g \in \Omega_{p}$ can be converted by the transformations $a(\cdot)$ and $b(\cdot)$ into any other gamble $g^{\prime} \in \Omega_{p}$. Using the riskless gamble $g_{0}=(0, p, 0)$ as origin, by means of the transformations $a(\cdot), b(\cdot)$, and $c(\cdot)$ applied to $g_{0}$ one gets a new risky gamble characterized by the triple $(a, b, c)$. Thereby, $a$ specifies gamble's $(a+b, p, b-$ $a p / q$ ) expected regret, $b$ gives this gamble's expectation, and 
$c$ states the number of independent repetitions of the gamble. Repetition multipies both expectation and expected regret.

Coombs and Huang presume that the perceived risk of a gamble $g \in \Omega_{p}$ characterized by $(a, b, c)$ is completely determined by these variables. As measure of risk of a gamble $g=(a, b, c)$ they propose the distributive model

$R(g):=[\alpha(a)+\beta(b)] \gamma(c)$,

where $\alpha(\cdot), \beta(\cdot)$, and $\gamma(\cdot)$ denote real-valued functions. Heuristically $\alpha, \beta$, and $\gamma$ correspond to three psychophysical functions for the subjective effects on perceived risk of the corresponding transformations $a(\cdot), b(\cdot)$, and $c(\cdot)$, respectively, on gambles. Empirical studies of Coombs and Huang $[23,24]$ showed that the function $\alpha(\cdot)$ may reasonably assumed to be strictly increasing, and $\beta(\cdot)$ stricty decreasing. The function $\gamma(\cdot)$ simultaneously intensifies the effect of $\alpha(\cdot)$ and $\beta(\cdot)$.

Coombs and Huang's measure of risk obviously is of low practical importance. It is tailored to a very special range of definition and Coombs and Huang do not say anything on how to specify the functions $\alpha(\cdot), \beta(\cdot)$, and $\gamma(\cdot)$. However, Coombs and Huang were mainly interested in whether perceived risk has the structure of the simple distributive model and, if so, what might be said further about the functions involved. As an empirical result of their study it can be noted that the risk of a gamble $g=(a, b, c)$ is perceived the higher the greater its variance and the lower the greater is its expectation. This follows from the above monotonicity properties.

\section{Pollatsek and Tversky's theory of risk}

Pollatsek and Tversky [52] were the first to investigate the perception of risk from the rigorous standpoint of measurement theory. Their theory is formulated in terms of a set $S$ of real-valued random variables, interpreted as gambles with (arbitray numbers of) monetary outcomes. This set $S$ is assumed to be closed with respect to the sum operation 0 of random variables. The key concept of Pollatsek and Tversky's theory of risk is a binary relation on $S$ denoted by $\succsim$ which is assumed to be strictly complete and transitive. For $\tilde{x}, \tilde{y} \in S, \tilde{x} \succsim \tilde{y}$ states that $\tilde{x}$ is at least as risky as $\tilde{y}$.

Pollatsek and Tversky require their risk ordering $\succsim$ to satisfy three different axioms, an independence axiom ("Cancellation") and two more technical axioms ("Solvability" and "Archimedian"). In a first representation theorem, they show that, if these axioms hold, there exists a realvalued function, $R$, defined on $S$, such that for any $\tilde{x}, \tilde{y} \in S$

$\tilde{x} \succsim \tilde{y} \quad \Longleftrightarrow \quad R(\tilde{x}) \geq R(\tilde{y})$

This risk measure is additive in the sense that for all $\tilde{x}$, $\tilde{y} \in S$

$R(\tilde{x} \circ \tilde{y})=R(\tilde{x})+R(\tilde{y})$,

and it is unique up to positive linear-homogeneous transformations. Introducing three additional assumptions about the risk ordering, two transformation axioms and a continuity axiom, Pollatsek and Tversky succeed in proving that there exists a unique real number $\theta, 0<\theta \leq 1$, such that for any $\tilde{x} \in S$ with expectation $E(\tilde{x})$ and variance $\operatorname{var}(\tilde{x})$ its risk $R(\tilde{x})$ is given with
$R(\tilde{x})=\theta \operatorname{var}(\tilde{x})-(1-\theta) E(\tilde{x})$

(Pollatsek and Tversky [52, Theorem 2, pp. 546-547]).

Thus, according to Pollatsek and Tversky's theory of risk, the risk ordering is generated by a linear combination of expectation and variance. The risk of any gamble with known expectation and variance can be readily computed, once a single parameter, $\theta$, is determined. Its value specifies the relative contribution of the expectation and the variance to the riskiness of that lottery. This value can be easily calculated from a single judgement of risk-equality between two different distributions. It should be noted that the risk measure of Pollatsek and Tversky can take on negative values and that degenerate distributions, i.e., distributions where a single value is obtained with probability one, may well have non-zero risk.

Subsequent empirical research has shown that the risk measure of Pollatsek and Tversky is not adequate. Coombs and Bowen [22] clearly have demonstrated that, despite the fact that perceived risk is indeed affected by both expectation and variance of a gamble, they alone are insufficient to determine risk. They found, by using transformations that let expectation and variance unchanged, that subjects detect differences in risk between gambles that have the same mean and variance. Actually, risk varied systematically with the skewness of a gamble. See Sect. 10 for this and other stylized facts derived from a number of empirical studies.

\section{Luce's measures of risk}

Ensuing approaches to the problem of risk measurement, consistently, concentrated on the problem how certain transformations of choice alternatives affected people's perceptions of their riskiness. These transformations included rescaling, i.e., multiplying all outcomes by a positive constant, as well as translation, i.e., adding a (positive or negative) amount to all outcomes (cf. Coombs and Bowen [22], Coombs and Huang [23, 24], and Coombs and Lehner [25]). Luce $[41,42]$ took up this approach by deriving risk measures from functional equations characterizing the effect of rescaling on perceived risk.

Luce [41] supresses the random variable notation and associates risk with densities. The set of all densities obviously is closed with respect to rescaling. If $f=f_{\tilde{x}}$ denotes the density function of a random variable $\tilde{x}$ then for the density $f_{\alpha \tilde{x}}$ of the transformed random variable $\alpha \tilde{x}$ holds

$f_{\alpha \tilde{x}}(x)=(1 / \alpha) f_{\tilde{x}}(x / \alpha)$

where $\alpha \tilde{x}$ denotes the random variable gained from $\tilde{x}$ through rescaling by a scale factor $\alpha>0$, i.e., through multiplying $\widetilde{x}$ by a positive real constant $\alpha$.

Luce presumes that the risk $R\left(f_{\alpha \tilde{x}}\right)$ of a density $f_{\alpha \tilde{x}}$ is some function of the risk $R\left(f_{\tilde{x}}\right)$ of the density $f_{\tilde{x}}$ and of $\alpha$. In his paper, he explores the two simplest possibilities, namely, that their effects are additive and that they are multiplicative. In a first assumption, Luce assumes that there is a strictly increasing function $S$ with $S(1)=0$ such that for all density functions $f_{\tilde{x}}$ and all real $\alpha>0$

$R\left(f_{\alpha \tilde{x}}\right)=R\left(f_{\breve{x}}\right)+S(\alpha)$. 
In his second assumption concerning the structure of risk, Luce assumes that there is an increasing function $S$ with $S(1)=1$ such that for all density functions $f_{\tilde{x}}$ and all real $\alpha>0$

$R\left(f_{\alpha \tilde{x}}\right)=S(\alpha) R\left(f_{\tilde{x}}\right)$.

A second class of assumptions concerns the nature of the aggregation of a density into a single number characterizing its risk. In a first assumption, Luce assumes that the density undergoes a pointwise transformation and then is integrated. More specifically, he assumes that there is a non-negative function $T$, with $T(0)=0$, such that for all density functions $f$

$R(f)=\int_{-\infty}^{+\infty} T(f(x)) d x$.

In a second assumption, Luce supposes that there is some transformation of the random variable itself and $R$ is the expectation of the resulting variable. More specifically, he assumes that there is a function $T$ such that for all densities $f$

$R(f)=\int_{-\infty}^{+\infty} T(x) f(x) d x=E(T(\tilde{x}))$

Combining each structural assumption with each aggregational, Luce gets four different functional forms of risk measures. The parameters of these functional forms depend on the subject's risk perception and are to be determined such that they fit this perception best. Combining the first aggregation rule (24) with the additivity assumption (22) leads to

$R_{1}(f)=-A \int_{-\infty}^{+\infty} f(x) \log f(x) d x+B$

with $A>0$ and $B \geq 0$. Combining it with the multiplicativity assumption (23) leads to

$R_{2}(f)=A \int_{-\infty}^{+\infty} f(x)^{1-\theta} d x$

with $A>0$ and $\theta>0$. With both measures, $R_{1}$ and $R_{2}$, the risk of a random variable $\tilde{x}$ is expressed by an integral of a certain non-linear transform of its density. The risk measure $R_{1}$, obviously, is an affine transformation of the Shannon entropy (see Sect. 2). In both measures, no difference is made between potential losses and potential gains of $\tilde{x}$.

Combining the second aggregation rule (25) with the additivity assumption yields

$$
\begin{aligned}
R_{3}(f)= & B_{1} \int_{0}^{\infty} f(x) d x+B_{2} \int_{-\infty}^{0} f(x) d x \\
& +A E(\log |\tilde{x}|),
\end{aligned}
$$

where $B_{1}, B_{2}$, and $A$ are real numbers, $A>0$. With this measure, the risk of a random variable $\tilde{x}$ is quantified by a linear combination of the expectation of the $\log$-transform of $\tilde{x}$, the probability of positive outcomes, and the probability of negative outcomes.

Combining the second aggregation rule (25) with the multiplicativity assumption yields

$R_{4}(f)=A_{1} \int_{0}^{\infty} x^{\theta} f(x) d x+A_{2} \int_{-\infty}^{0}|x|^{\theta} f(x) d x$

where $\theta$ is a real number, $\theta>0$, and

$A_{1}=(\theta+1) \int_{0}^{1} T(x) d x$

and

$A_{2}=(\theta+1) \int_{-1}^{0} T(x) d x$.

With the measure $R_{4}$, the risk of a random variable $\tilde{x}$ is represented by a linear combination of the conditional expectation of positive outcomes and the conditional expectation of negative outcomes, where all outcomes are raised to some power $\theta$. An important feature of the risk measures $R_{3}$ and $R_{4}$ is that gains and losses are treated separately and in a different manner. In the measure $R_{4}$, the "chance component" of $\tilde{x}$, i.e. the possible gains, and its pure "risk component", i.e. the possible losses, combine clear-cut additively.

Luce leaves the question of the reasonableness of these forms to empirical investigation. Nevertheless, he remarks that many psychologists believe that the risk of a gamble that is repeated $n$ times is less than $n$ times the risk of the gamble played once. This is known to hold only for the risk measure $R_{3}$ and fails for the others. However, the risk measure $R_{3}$ suffers, as well as the risk measure $R_{4}$, from another drawback. It increases with $a$ for positive uniformly distributed random variables with constant range $b-a$. For some people, this property is highly counter-intuitive. In fact, there is empirical evidence that risk decreases if a positive constant is added to all outcomes of a gamble (see Sect. 10).

There are two fundamental problems with Luce's structural and aggregational assumptions. A first problem concerns the additivity assumption (22). This assumption obviously implies that a gamble having zero risk is transformed by any change of scale into one with non-zero risk. Rescaling by any positive factor $\alpha<1$ leads to negative risk, whereas risk is increased by rescaling with any $\alpha>1$. This argument favours the multiplicativity assumption (23) and thus the risk measures $R_{2}$ and $R_{4}$.

A second fundamental problem concerns the first aggregation rule (24). This aggregation rule leads to risk measures which are translation invariant or location free. Thereby, a risk measure $R$ is called translation invariant or location free if and only if

$R\left(f_{\tilde{x}+\beta}\right)=R\left(f_{\tilde{x}}\right)$

where $f_{\tilde{x}+\beta}$ denotes the density of the transformed random variable $\tilde{x}+\beta$ gained from $\tilde{x}$ through translation by $\beta$, i.e., by adding a real constant $\beta$. Translation invariance of risk measures of type (24) is immediately shown by 


$$
\begin{aligned}
R\left(f_{\tilde{x}+\beta}\right) & =\int T\left(f_{\tilde{x}+\beta}(x)\right) d x=\int T\left(f_{\tilde{x}}(x-\beta)\right) d x \\
& =\int T\left(f_{\tilde{x}}(x)\right) d x=R\left(f_{\tilde{x}}\right) .
\end{aligned}
$$

Translation invariance of risk measures of type (24) implies that the risk measures $R_{1}$ and $R_{2}$, for any shift family of distributions, depend only on the range and are independent of the location of a random variable. It follows, e.g., that gambles with a uniform distribution of the same range $b-a$ are, in the sense of $R_{1}$ and $R_{2}$, equally risky irrespectively of their location. Again, this property can be regarded as highly counter-intuitive. In fact, it can be deduced from the empirical evidence mentioned above that risk depends on the location of a gamble (see Sect. 10).

It should be noted that also most of the naive risk measures reviewed in Sect. 2 are translation invariant. This holds in particular for the most important risk measures used in finance, namely variance (3), standard deviation (4), and lower semivariance (7). Risk of a random variable is measured independent of its location. In contrast to this, Coombs' distributive risk model (17) as well as Pollatsek and Tversky's risk measure (20), are not location free. Both risk measures decrease if a positive constant is added to all outcomes of a gamble. For a short discussion of the issue of translation invariance of risk measures, again, see Sect. 10.

E.U. Weber $[62,63]$ investigated Luce's four assumptions (22) through (25) and the properties of the risk measures implied. Empirical evidence against translation invariance of perceived risk (see E.U. Weber [62]), first, led her to reject assumption (24) and therefore the risk measures (26) and (27). Then she pointed out (see E.U. Weber [63]) that the risk measure (28) can be ruled out because of its unreasonable behavior in the neighborhood of zero. Obviously, this measure approaches negative infinity if, e.g., any positive gamble with uniform distribution is rescaled by a factor $\alpha>0$ converging to zero.

Revising and extending Luce's model (29), Luce and E.U. Weber [44] presented a new axiomaticly based risk model, called conjoint expected risk (CER). Like Pollatsek and Tversky [52], Luce and E.U. Weber start from an arbitrary set $\mathscr{F}$ of real-valued random variables, interpreted as gambles with (arbitray numbers of) monetary outcomes, and assume that the decision maker involved has a binary risk ordering, $\succsim$, on $\mathscr{G}$. Then the CER model is derived from a certain system of axioms or assumptions on $\succsim$.

First, two axioms are presented which are purely technical and could be omitted if the authors assumed that the domain of the risk measure consists of all possible random variables. Another axiom simply postulates that the risk measure is well behaved near 0 . A further axiom requires that the risk ordering fulfills certain assumptions implying the expectation principle, i.e., transitivity, continuity, and independence, well known from expected utility literature. These four axioms do not offer any special insight into what is special about the CER measure. It is a final fifth axiom which shapes the risk ordering.

This axiom consists of four requirements on $\succsim$. Let $\tilde{x}$ and $\tilde{y}$ be two random variables in $\mathscr{F}$ which, both, can take on only positive outcomes or, both, can take on only negative outcomes and let $a, b, b^{\prime}$, and $b^{\prime \prime}$ be positive real numbers.
Then, the first requirement of this axiom is the independence condition

$\tilde{x} \succsim \tilde{y} \Longleftrightarrow a \tilde{x} \succsim a \tilde{y}$,

and

$a \tilde{x} \succsim b \tilde{x} \quad \Longleftrightarrow a \tilde{y} \succsim b \tilde{y}$.

Condition (34) states that a change in scale does not change the risk ordering between random variables which, both, can take on only positive outcomes or, both, can take on only negative outcomes. The second part (35) of the independence condition says that if, for any random variable which can take on only positive or only negative values, one scale is perceived as at least as risky than onother scale, then the same ordering holds for any other random variable which can take on only positive or only negative values, respectively.

The second condition states that the ordering induced by independence on the positive reals $\mathbb{R}^{+}$is the ordinary ordering $\geq$, i.e.

$a \tilde{x} \succsim b \tilde{x} \Longleftrightarrow a \geq b$.

Assuming independence, this condition says that the riskiness of gambles which can take on only positive or only negative outcomes is an increasing function of the scale value. Thus, e.g., the gamble $\tilde{x}=(\$ 15, .5 ; \$ 1)$ is perceived to be less risky than the gamble $4 \tilde{x}=(\$ 60, .5 ; \$ 4)$.

As a third requirement a condition of restricted solvability is introduced which states that for any two random variables $\tilde{x}$ and $\tilde{y}$ which, both, can take on only positive values or, both, can take on only negative values there exists a positive real number $b$ such that

$b^{\prime} \tilde{y} \succsim a \tilde{x} \succsim b^{\prime \prime} \tilde{y} \quad \Longrightarrow \quad b \tilde{y} \sim a \tilde{x}$.

This solvability condition says that perceived risk is a continuous function of scale changes. Note, that this solvability condition is different but related to the standard continuity assumption which is part of the axioms which imply the expectation principle.

As the fourth requirement of this axiom an Archimedean condition is introduced which states that for any two random variables $\tilde{x}$ and $\tilde{y}$ which, both, can take on only positive outcomes or, both, can take on only negative outcomes there exists a positive real number $a$ such that

$\tilde{x} \succ \tilde{y} \quad \Longrightarrow \quad a \tilde{y} \succsim \tilde{x}$

This condition says that given two gambles $\tilde{x}$ and $\tilde{y}$ where $\tilde{x}$ is riskier than $\tilde{y}$, then, by means of a sufficiently large scale transformation, $\tilde{y}$ can be transformed into a gamble that is at least as risky as $\tilde{x}$.

Based on these axioms on the risk ordering $\succsim$, Luce and E.U. Weber [44] prove that $\succsim$ can numerically be represented through the CER-model $\widetilde{R_{C E R}}(\tilde{x})$ which, for any (discrete or continuous) random variable $\tilde{x} \in \mathscr{Y}$, is given by

$$
\begin{aligned}
R_{C E R}(\tilde{x})= & B_{1} \int_{-\infty}^{0} d F_{\tilde{x}}(x)+B_{2} \int_{0}^{\infty} d F_{\tilde{x}}(x) \\
& +B_{3} \int_{0}^{0} d F_{\tilde{x}}(x)+A_{1} \int_{-\infty}^{0}|x|^{\theta_{1}} d F_{\tilde{x}}(x) \\
& +A_{2} \int_{0}^{\infty} x^{\theta_{2}} d F_{\tilde{x}}(x)
\end{aligned}
$$


where $B_{i}, A_{i}$, and $\theta_{i}$ are scaling constants, $\theta_{i}>0$, and $F_{\widetilde{x}}$ denotes the distribution function corresponding to $\tilde{x}$.

As a result, according to the CER-model, perceived risk of a "gamble" $\tilde{x}$ can be quantified by a linear combination of the probability of negative outcomes, the probability of positive outcomes, and the probability of the zero outcome as well as the conditional expectation of negative outcomes raised to some power $\theta_{1}$ and the conditional expectation of positive outcomes raised to some power $\theta_{2}$. As Luce's measure $R_{4}$, the measure $R_{C E R}$ evaluates gains differently from losses and the "chance component" of a "gamble" and its pure "risk component" combine additively. But, contrary to $R_{4}$, the probabilities to win, to lose and to break even are additionally part of this risk measure. For gambles with only positive or only negative outcomes the CER-model is equivalent to $R_{4}$. Therefore, it suffers from the same behavioral problems as pointed out above for $R_{4}$. The high number of scaling constants poses an additional challenge for a reliable assessment of the risk measure $R_{C E R}$.

It should be noted that, in general, the scaling constants $A_{i}$ and $B_{i}$ of the CER-model (39) can take on negative or positive values, depending on the decision maker's risk ordering. For a particular individual, these scaling constants can be easily estimated from a sample of observed judgements of perceived risk. Based on their perceived risk judgements, E.U. Weber [64] estimated the parameters of the CER-model for several individuals. For all of these individuals she found $A_{1}$ and $B_{1}$ to be positive as well as $A_{2}$ and $B_{2}$ to be negative. In such cases, the probability of positive outcomes of a gamble as well as their conditional expectation reduce the gamble's perceived risk and the risk measure (39) can take on negative values when the positive outcome contributions outweigh the negative outcome contributions.

It is interesting to think about the system of axioms on which the CER-model is founded. All axioms make intutive sense, and, in addition, do not appear to be so strong. Nevertheless, these axioms imply a pretty restrictive set of risk measures, quite different from what we will come to know in the next sections.

\section{Sarin's measures of risk}

The purpose of Sarin's paper [53] is to extend Luce's risk measures to obtain risk measures that are empirically more reasonable. Therefore, Sarin starts from the overwhelming empirical observation that the risk of a gamble appears to decrease when all possible outcomes are improved by a constant, i.e., when a positive constant is added to all outcomes of a gamble.

Sarin's first assumption concerns the risk of the density $f_{\tilde{x}+\beta}$ belonging to the transformed random variable $\tilde{x}+\beta$. He assumes that $R\left(f_{\tilde{x}+\beta}\right)$ is a multiplicative function of $R\left(f_{\tilde{x}}\right)$ and $\beta$. More specifically, it is assumed that there is a strictly monotonic function $S$ with $S(0)=1$ such that for all density functions $f_{\tilde{x}}$ and all real $\beta>0$

$R\left(f_{\tilde{x}+\beta}\right)=S(\beta) R\left(f_{\tilde{x}}\right)$.

Thereby, without being explicitly stated, it is assumed that $R\left(f_{\tilde{x}+\beta}\right)$ decreases as $\beta$ increases. For non-negative risk measures this implies that $S(\cdot)$ is strictly decreasing.
As indicated in the last section, Luce's first aggregational assumption (24) implies risk measures which are translation invariant. Holding such risk measures to be empirically not reasonable, Sarin therefore, in his second assumption, requires that the expectation principle (25) be used to aggregate densities into single numbers.

From these two assumptions Sarin derives the risk measure

$R(f)=\int_{-\infty}^{+\infty} K e^{c x} f(x) d x=K E\left(e^{c \tilde{x}}\right)$,

with real constants $K>0, c<0$, or $K<0, c>0$. Through this measure, risk of a random variable $\tilde{x}$, essentially, is represented by the expectation of its exponential transform.

As, for example, implicitly stated in Luce's assumption (24), it seems sensible to assume risk measures to be nonnegative. This implies for Sarin's risk measure $c<0$ and $K>0$. Evidently, this risk measure gives higher weight to a gamble's potential losses than to its potential gains. Because of assumption (40), Sarin's risk measure does not suffer the last critizism of Luce's risk measures, it is not location free and, in particular, decreases under translations with increasing $\beta$. Furthermore, it can be easily shown that it increases with the scale factor $\alpha$ under rescaling.

Weber [68], contrary to Sarin advocating translation invariance of risk judgements, presents an extension of Sarin's risk measure (41). To make this measure location free he suggests, first, to "normalize" gambles by substracting their respective expected values, i.e., to transform all random variables $\tilde{x}$ to the "normalized" variables

$\tilde{x}^{\prime}=\tilde{x}-E(\tilde{x})$.

The "normalized" random variables all have zero mean and reflect the risk of the original distributions with reference to their expectation. Thereby, the expectation serves as a target outcome such that every outcome whose value is smaller than the expectation is viewed as undesirable or risky, while outcomes with values as large as the expectation are desirable or nonrisky.

Weber's location free variant of Sarin's risk measure (41) is then given with

$R^{\prime}(f)=\int_{-\infty}^{+\infty} K e^{c(x-E(\tilde{x}))} f(x) d x=K E\left(e^{c \tilde{x}^{\prime}}\right)$,

with real constants $K>0, c<0$. Through this measure, risk of a random variable $\tilde{x}$, essentially, is represented by the expectation of the exponential transform of its normalized version $\tilde{x}^{\prime}$. Weber points out that for normally distributed random variables the measure (43) and the variance (3) yield the same risk ordering of lotteries. In Sect. 9 will be shown that (43) can also be derived using a quite different way of arguing.

Sarin's risk measure (41) as well as Luce's risk measures $R_{3}$ and $R_{4}$ use the expectation principle for aggregating densities into single numbers. This principle implies that resulting risk measures are linear in probabilities. I.e., if, for $0<\theta<1$, the gamble with $\theta$ chance of obtaining density 
$f$ and $(1-\theta)$ chance of obtaining density $f_{o}$ is denoted by $\theta f+(1-\theta) f_{o}$, we have

$R\left(\theta f+(1-\theta) f_{o}\right)=\theta R(f)+(1-\theta) R\left(f_{o}\right)$.

Thus, if $f$ and $g$ are judged to be equally risky, then all the gambles $\theta f+(1-\theta) f_{o}$ and $\theta g+(1-\theta) f_{o}$ will also be judged equally risky. In other words, if the expectation principle is used for aggregating densities into risk numbers, then, for any given distribution $f_{o}$, the risk function is, between $f_{o}$ and any other distribution $f$, independent of $f$ as long as $f$ belongs to a set of distributions with some given risk level.

It is well-known that linearity in probabilities is a pretty strong requirement when preferences are modelled. In decision theory under risk quite a number of new theories has been developed to overcome this strong requirement implied by the expectation principle (for an overview see Weber and Camerer [69]). In the spirit of the generalization of expected utility theory by Machina [45], Sarin generalizes Luce's expectation assumption (25) to

$R(f)=\int_{-\infty}^{\infty} T(x) f(x) d x+\frac{1}{2}\left[\int_{-\infty}^{\infty} S(|x|) f(x) d x\right]^{2}$.

Thereby, $T(\cdot)$ is a real function and $S(\cdot)$ is that strictly monotonic function the existence of which is postulated in Luce's assumptions (22) and (23), as well as in Sarin's assumption (40). In this generalized expectation form of a risk measure the "local" risk function depends on the gamble. As a consequence, even if two densities $f$ and $g$ are equally risky, $\theta f+(1-\theta) f_{o}$ and $\theta g+(1-\theta) f_{o}$ may not have equal risk.

Sarin points out that the generalized expectation assumption (45) can, just as Luce's expectation assumption, be combined with different structural assumptions to derive alternative measures of risk. Combining the generalized expectation assumption, in turn, with Luce's structural assumptions Sarin gets two new families of risk measures. Combining it with the additivity assumption (22) he obtains the risk measure

$$
\begin{aligned}
R(f)= & B_{1} \int_{0}^{\infty} f(x) d x+B_{2} \int_{-\infty}^{0} f(x) d x \\
& +A E(\log |x|)-\frac{1}{2} A^{2} \operatorname{var}(\log |x|)
\end{aligned}
$$

with some constants $B_{1}, B_{2}$, and $A>0$. Combining it with the multiplicativity assumption (23) he receives the risk measure

$$
\begin{aligned}
R(f)= & B_{1} \int_{0}^{\infty} x^{\theta} f(x) d x+B_{2} \int_{-\infty}^{0}|x|^{\theta} f(x) d x \\
& +\frac{1}{2}\left(\frac{\theta}{\theta-1}\right) E\left(|x|^{2 \theta}\right)-\frac{1}{2} \operatorname{var}\left(|x|^{\theta}\right) .
\end{aligned}
$$

It should be noted that in the generalized risk measures (46) and (47) of Sarin as well as already in Luce's risk measures (28) and (29) potential gains and potential losses are treated separately. This seems to be in accordance to observed differential preference attitudes towards gains and losses. Sarin stresses the fact that empirical data will be needed to test the validity of the generalized expectation principle and the corresponding generalized risk measures.

\section{Fishburn's measures of pure risk}

Various empirical studies have demonstrated that preference attitudes towards gambles exhibit striking differences in the loss and the gain regions (cf. Fishburn and Kochenberger [31], Hershey et al. [33], Kahneman and Tversky [35], Laughhunn et al. [40], Payne et al. [50, 51]). Although the association of preferences between probability distributions with the distributions' risks is far from being clear, it seems reasonable to assume that these differences also play an important role in risk assessments. Furthermore, experiments with monetary gambles have convincingly shown the salience of loss probability and loss amount on perceived risk (cf. Aschenbrenner [5], Coombs and Lehner [25], Payne [49], Slovic [57]). Based on these observations, Fishburn, in his Foundations of Risk Measurement [29, 30], developed theories of risk in which gains and losses are treated separately. In the first part of his study, Fishburn focusses on risk as probable loss, i.e. on what is usually called pure risk. In the second part, measures of risk are proposed that include effects of gains on perceived risk.

In the first part of his study, Fishburn is guided by the conventional wisdom that risk is a chance of something bad happening, that risk arises from the possibility of undesired outcomes. As Pollatsek and Tversky, Fishburn's theory of risk is based on a binary risk relation $\succsim$, "is at least as risky as", defined on a set of probability distributions of some outcome values. From the standpoint of measurement theory, this relation is to be represented by a numerical risk measure. Similar to the way Pollatsek and Tversky derive their risk measure, Fishburn's measures are a direct consequence of the axioms the risk relation is fulfilling.

In the spirit of most of the classical risk measures used in finance and of Luce's assumption 24, Fishburn implicitly adopts the position that risk judgements are location free. Therefore he presumes that some target outcome can be identified so that every outcome whose value is smaller than the value of the target is viewed as undesirable or risky, while outcomes with values as large as the target's are desirable or nonrisky. Without loss of generality, for convenience, the value of this target outcome is set at zero. Fishburn regards a distribution as having zero risk if and only if it has no chance of delivering a outcome below zero.

More specifically, Fishburn starts from the assumption that all possible outcomes are ordered by preference and represented numerically so that one outcome is preferred to another if and only if the former has a larger value. Let $X$ denote the (non-empty) set of all possible outcome values. Non-zero outcome values are partitioned into undesirable and desirable subsets as $X^{-}:=\{x \in X \mid x<0\}$ and $X^{+}:=\{x \in X \mid x>0\}$. The outcomes in $X^{-}\left(X^{-} \neq \emptyset\right.$ by assumption) are referred to as losses, and the outcomes in $X^{+}$as gains.

Probability distributions of $X$ are defined on the $\sigma$ algebra of subsets of $X$ generated by all singletons and "intervals" of outcomes. With regard to its pure risk every probability distribution of $X$ is characterized by the pair $(\alpha, p)$, where the parameter $\alpha, 0 \leq \alpha \leq 1$, gives the loss probability of that distribution and $p$ is its conditional distribution, given a loss. The pair $(\alpha, p)$ is to be interpreted as a two-dimensional measure that yields the probability of a 
subset $Y$ of $X^{-}$as $\alpha p(Y)$. Then, the binary risk relation $\succsim$ is applied to the set

$A:=[0,1] \times P^{-}:=\left\{(\alpha, p) \mid 0 \leq \alpha \leq 1, p \in P^{-}\right\}$,

where $P^{-}$is the set of all probability measures $p$ with $p\left(X^{-}\right)=1$. By identifying every outcome value $x \in X^{-}$ with $(1, p)$ where $p(\{x\})=1$, this set contains all deterministic losses.

The risk relation $\succsim$ defined on $A$ is assumed to be a weak order, i.e. strictly complete and transitive. Then, for a first basic representation theorem, Fishburn uses four axioms. Among these a continuity axiom and an axiom that states that for every measure in $P^{-}$, some deterministic loss is at least as risky as the measure. Another axiom states that risk increases as the loss probability increases for fixed $p$, another one that worse outcomes entail greater risks. The latter axioms seem reasonable in view of the common perception that risk increases as bad outcomes become more probable, and as probable bad outcomes get worse. In his first basic representation theorem, Fishburn shows that there is a non-negative real-valued function $\rho$ on $A$ with

$(\alpha, p) \succsim\left(\alpha^{\prime}, p^{\prime}\right) \quad \Longleftrightarrow \rho(\alpha, p) \geq \rho\left(\alpha^{\prime}, p^{\prime}\right)$

which has $\rho(\alpha, p)=0$ if and only if $\alpha=0$, and is continuous and increasing in $\alpha$.

Three additional axioms yield what Fishburn calls $\alpha-p$ separability, namely the existence of real-valued functions $\rho_{1}$ on $[0,1]$ and $\rho_{2}$ on $P^{-}$such that

$\rho(\alpha, p)=\rho_{1}(\alpha) \cdot \rho_{2}(p)$

Thereby, the function $\rho_{1}$ is continuous and increasing in $\alpha$ with $\rho_{1}(0)=0$, and the functional $\rho_{2}$ is, restricted to onepoint-distributions $p_{x}$ over $X^{-}$, increasing as $x$ decreases.

Certain combinations of additional axioms, then, yield special forms of risk measures of the multiplicative separable type (50). Fishburn axiomizes risk measures of type

$\rho(\alpha, p)=\rho_{1}(\alpha) \int_{X^{-}} \rho_{2}(x) d p(x)$,

$\rho(\alpha, p)=\alpha \int_{X^{-}} \rho_{2}(x) d p(x)$,

and

$\rho(\alpha, p)=\rho_{1}(\alpha) \int_{X^{-}}|x|^{\theta} d p(x)$,

where $\theta$ is a real parameter, $\theta>0$. With all of these types of risk measueres, each outcome $x$ is identified with the one-point measure $p_{x}$ and integration is Lebesque-Stieltjesintegration.

In a further theorem, Fishburn gives conditions for the representation of $\succsim$ by a risk measure of the expectation type

$\rho(\alpha, p)=\int_{X^{-}} \rho(\alpha, x) d p(x)$.

This corresponds to the expectation principle (25) used by Luce and Sarin as aggregational assumption.
These families of risk measures contain some of the naive risk measures listed in Sect. 2 as special cases. In the family (53) of risk measures, the choice $\rho_{1}(\alpha) \equiv \alpha$ together with $\theta=1$ yields the expected value of loss (8) of a random variable; the choice $\rho_{1}(\alpha) \equiv \alpha$ together with $\theta=2$ leads to the lower semivariance (7) of a mean-centered random variable. Furthermore, the family (53) of risk measures with $\rho_{1}(\alpha) \equiv \alpha$ is contained in Stone's family (10), if one chooses there $p(F)=q(F)=0$. The risk measures (52) and (53) obviously are special cases of the more general familiy (51). With the choice $\rho_{1}(\alpha) \equiv \alpha$, the family (51) yields (52), with the choice $\rho_{2}(x)=|x|^{\theta}$ it yields (53). With the choice $\rho_{1}(\alpha) \equiv \alpha$, the family (53) leads to the risk measures (12) which Fishburn considered in his earlier paper [28].

\section{Fishburn's measures of speculative risk}

In the second part of his Foundations of Risk Measurement [29], Fishburn considers measures of risk that explicitly include effects of gains on perceived risk. He adopts the position that increased gains reduce the risk of fixed probable losses without completely negating this risk. Thus he focusses on what is usually called speculative risk. Speculative risk measures, generally, are to incorporate the consensus that risk increases as loss probability or amount increases, and that greater gains as well as greater gain probabilities reduce perceived risk. As Fishburn's theory of pure risk, his theory of speculative risk is based on a binary risk relation $\succsim$, "is at least as risky as", defined on a set of probability distributions of some outcome values. Again, this relation is to be represented by a numerical risk measure.

As in his first study, Fishburn starts from a non-empty set $X$ of numerical outcome values that includes a nonrisky target outcome. Without loss of generality, the value of that target outcome is set at zero. As above, the set of all non-zero outcomes is partitioned into an undesirable subset $X^{-}$of losses and a desirable subset $X^{+}$of gains, each of which is assumed non-empty. Furthermore, it is assumed that outcome preference increases in $x$.

Again, probability distributions of $X$ are defined on the $\sigma$-algebra of subsets of $X$ generated by all singletons and "intervals" of outcomes. With regard to its speculative risk every probability distribution of $X$ is characterized by the quadrupel $(\alpha, p ; \beta, q)$, where, as above, the parameter $\alpha, 0 \leq$ $\alpha \leq 1$, gives the loss probability of that distribution and $p$ is its conditional distribution, given a loss. The parameter $\beta, 0 \leq \beta \leq 1$, gives the gain probability of that distribution and $q$ is its conditional distribution, given a gain. Hence, the probability for the target-outcome $\{0\}$ equals $1-\alpha-$ $\beta$. The quadrupel $(\alpha, p, \beta, q)$ is to be interpreted as a fourdimensional measure that yields the probability for a subset $Y$ of $X^{-}$as $\alpha p(Y)$, and the probability for a subset $Z$ of $X^{+}$as $\beta q(Z)$.

Then, the risk relation is applied to the set

$$
\begin{gathered}
B=\{(\alpha, p ; \beta, q) \mid \alpha, \beta \geq 0, \alpha+\beta \leq 1, \\
\left.p \in P^{-}, q \in P^{+}\right\},
\end{gathered}
$$

where $P^{-}$is defined as above, and $P^{+}$is the set of all probability measures $q$ with $q\left(X^{+}\right)=1$. By identifying every outcome value $x \in X^{-}$with $(1, p)$ where $p(\{x\})=1$ and 
every outcome value $x \in X^{+}$with $(1, q)$ where $q(\{x\})=1$, this set contains all deterministic losses and gains.

Also in this generalized case, the risk relation $\succsim$ defined on $B$ is assumed to be a weak order. Then, Fishburn presents sufficient conditions for a basic representation of $\succsim$ by a non-negative real-valued function $R$ on $B$ that has

$R(\alpha, p ; \beta, q)=0 \quad \Longleftrightarrow \quad \alpha=0$

and satisfies, for all $(\alpha, p ; \beta, q),(\gamma, r ; \delta, s) \in B$

$$
\begin{aligned}
&(\alpha, p ; \beta, q) \succsim(\gamma, r ; \delta, s) \Longleftrightarrow \\
& R(\alpha, p ; \beta, q) \geq R(\gamma, r ; \delta, s) .
\end{aligned}
$$

It should be noted that, according to condition (56), there is no risk if and only if there is no chance of getting an undesirable outcome. Therefore, this condition rules out measures of risk that are additively separable in gains and losses in the sense of

$R(\alpha, p ; \beta, q)=R_{1}(\alpha, p)+R_{2}(\beta, q)$,

with a gain part $R_{2}$ and a loss part $R_{1}$.

Condition (56) has two interesting empirical implications. According to that condition, every sure loss $(\alpha=1)$ has non-zero risk and is therefore regarded as risky. In addition, every $50-50$ gamble of receiving the target outcome or some amount above the target outcome $(\alpha=0)$ has zero risk and is therefore regarded as riskless. Both properties are highly controversial, as can be verified in any class-room experiment.

For a first representation theorem, Fishburn uses five axioms. Among these are a continuity condition for loss and gain probabilities and an axiom which asserts some suitably bad outcome which is at least as risky as a given $p \in P^{-}$. The third axiom states the commitment to no risk when there is no chance of loss. The first two axioms assert monotonicity for gain and loss probabilities and for gains and losses, respectively. These axioms are in accordance with the conventional notion of speculative risk. In his representation theorem, Fishburn shows that there is a non-negative realvalued function $R$ on $B$ satisfying conditions (56) and (57) that is continuous and increasing in loss probability as well as continuous and decreasing in gain probability when the loss probability is positive.

In Fishburn's study a series of further axioms is formulated. Certain groups of axioms assure special types of risk measures. According to commitment (56), only such representations are considered which are multiplicatively separable in a risk-part quantifying an option's pure risk and a chance-part expressing an option's potential gain. In his approach, Fishburn essentially follows conventional conjoint measurement (cf. Krantz, Suppes, Luce, and Tversky [39]). The axiomatized types of risk measures include

$$
\begin{aligned}
R(\alpha, p ; \beta, q)= & \rho(\alpha, p) \tau(\beta, q) \\
R(\alpha, p ; \beta, q)= & {\left[\int_{X^{-}} \rho(\alpha, x) d p(x)\right]\left[\int_{X^{+}} \tau(\beta, y) d q(y)\right] } \\
R(\alpha, p ; \beta, q)= & {\left[\rho_{1}(\alpha) \int_{X^{-}} \rho_{2}(x) d p(x)\right] } \\
& \times\left[1-\tau_{1}(\beta) \int_{X^{+}} \tau_{2}(y) d q(y)\right]
\end{aligned}
$$

These types of risk measures get more and more specialized. The family (59) has the basic multiplicatively separable form. The second is a specialization using the expectation principle for the risk-part as well as for the chance-part. The third family (61) goes a step further by separating out the effects of loss and gain probabilities. Assuming that the indifference relation $\sim$ implied by the risk relation $\succsim$ is preserved under uniform rescaling of outcomes in his pure risk setting Fishburn, finally, arrives at the risk measure

$$
\begin{aligned}
R(\alpha, p ; \beta, q)= & {\left[\rho_{1}(\alpha) \int_{X^{-}}|x|^{\theta} d p(x)\right.} \\
& \times\left[1-\tau_{1}(\beta) \int_{X^{+}} \tau_{2}(y) d q(y)\right] .
\end{aligned}
$$

The central idea of all these families of speculative risk measures is that gains reduce risk in a proportional way that is independent of the particular $(\alpha, p)$ involved, unless $\alpha=0$, in which case there is no risk to be reduced (cf. Fishburn $[29$, p. 228]).

The risk measure (62) is the most specific risk measure axiomatized by Fishburn. For deducing this measure he refers to no less than 19 axioms. Fishburn [29, p. 242] himself doubts the importance of his axiomatization: "I wish I could say that the axiomatization for this measure was elegant, straightforward, and convincing, but this is clearly absurd in view of the number of axioms used ... and the tentative nature of some of them for perceived risk."

\section{Risk defined through utility}

In the literature reported so far, mixing measurement of perceived risk with preference measurement has been rather carefully avoided. Jia and Dyer [34] take a different approach. As with other authors before their objects of risk measurement are (discrete or continuous) random variables which are regarded as lotteries or gambles with (arbitrary number of) monetary outcomes. Like Fishburn, they believe that mere risk judgements are a "relative matter" and should be independent of location. Jia and Dyer therefore presume that the perceived riskiness of a lottery is evaluated with reference to a certain target level and advocate that the expected value of a lottery should serve as such a reference point for identifying relative gains and losses. Furthermore, Jia and Dyer believe that the expectation principle (25) should be used as aggregation rule.

Jia and Dyer [34] start from a convex set $\mathscr{C}$ of probability distributions over a nonempty set $X$ of possible outcomes. Each probability distribution is identified with the corresponding random variable $\tilde{x}$. To end up with a location free "standard measure of risk", Jia and Dyer first like Weber [68] (cf. Sect. 6) "normalize" gambles by substracting their respective expected values $E(\tilde{x})$. As mentioned above, the "normalized" variables

$\tilde{x}^{\prime}=\tilde{x}-E(\tilde{x})$.

all have zero mean and reflect the risk of the original distribution with reference to the target outcome $E(\tilde{x})$.

As domain of the risk ordering $\succsim$, then, Jia and Dyer take the set 
$\mathscr{\mathscr { V }}^{\circ}=\left\{\tilde{x}^{\prime} \mid \tilde{x}^{\prime}=\tilde{x}-E(\tilde{x}), \tilde{x} \in \mathscr{Q}\right\}$

of all normalized random variables induced by $\mathscr{D}$. Comparing the asymmetric part $\succ$ of the risk ordering with the decision maker's strict preference ordering $\succ_{p}$ on $\mathscr{D}^{\circ}$, they assume that there is an inverse relationship between $\succ$ and $\succ_{p}$ in the sense that for all $\tilde{x}^{\prime}, \tilde{y}^{\prime} \in \mathscr{X}^{\circ}$ holds

$\tilde{x}^{\prime} \succ \tilde{y}^{\prime} \Longleftrightarrow \tilde{x}^{\prime} \prec_{p} \tilde{y}^{\prime}$.

Supposing that the preference relation $\succ_{p}$ satisfies the axioms of the Expected Utility Model, Jia and Dyer define, for any $\tilde{x} \in \mathscr{D}$ their standard measure of risk by

$R(\tilde{x})=-E\left[u\left(\tilde{x}^{\prime}\right)\right]=-E[u(\tilde{x}-E(\tilde{x}))]$

where $u$ is the decision maker's von Neumann-Morgenstern utility function. As well as the utility function, $R$ is unique up to positive affine transformations. Under the assumptions (65), for this risk measure holds

$\tilde{x}^{\prime} \succ \tilde{y}^{\prime} \Longleftrightarrow R\left(\tilde{x}^{\prime}\right)>R\left(\tilde{y}^{\prime}\right)$.

Because the standard measure of risk is only unique up to positive affine transformations it can always be rescaled such that all degenerate lotteries have zero risk. This can be achieved by setting

$R^{\prime}(\tilde{x})=R(\tilde{x})-R(0)=R(\tilde{x})+u(0)$.

One advantage of the standard measure of risk is that it does not impose any restrictions on the set $\mathscr{D}$ of random variables on which the risk ordering is defined or the form of the decision maker's utility function $u$. Through its quite general definition, this measure of risk allows for a number of different risk measures thus giving less structure than the risk measures presented in the earlier sections.

The main advantage of the standard measure of risk is that it can be easily incorporated into a risk-value framework which allows the derivation of a measure of perceived risk. Jia and Dyer [34] show that if the axioms of the Expected Utility Model hold and if, in addition, a certain condition of risk independence is fulfilled the decision maker's expected utility $E[u(\tilde{x})]$ allows the decomposition

$E[u(\tilde{x})]=u(E(\tilde{x}))-\phi(E(\tilde{x})) R^{\prime}(\tilde{x})$,

where $\phi$ is a monotonically decreasing positive real function. This decompositon shows that the expected utility $E[u(\tilde{x})]$ of a risky random variable $\tilde{x}$ is equal to the utility of its expectation which is, because of the riskiness of $\tilde{x}$, reduced by an amount proportional to the rescaled standard measure of risk. Thereby, $\phi(E(\tilde{x}))$ can be interpreted as a trade-off factor between "value" $u(E(\tilde{x}))$ and "risk" $R^{\prime}(\tilde{x})$.

Jia and Dyer suggest to consider the "risk"-part of (69) as a measure of the "intensity of the risk effect on preference" and, therefore, to use it as a measure of perceived risk. As already mentioned in Sect. 5 , there is empirical evidence that subjects' perceived risk decreases if a positive constant is added to all outcomes of a lottery. For that reason, the tradeoff function $\phi$ should be decreasing.

Jia and Dyer's measure of perceived risk is given with

$R^{*}(\tilde{x})=\phi(E(\tilde{x})) R^{\prime}(\tilde{x})=\phi(E(\tilde{x}))[R(\tilde{x})-R(0)]$,

where $\phi$ is a monotonically decreasing positive real function. This measure of perceived risk keeps the appealing properties of the standard measure of risk (66). It is zero for degenerate lotteries, i.e. for lotteries with a single sure outcome, and adding a positive constant to all outcomes of a given lottery decreases its risk.

The risk measure (66) obviously depends on the decision maker's utility function. If one takes, e.g., $u$ to be a linear plus exponential utility model, $u(x)=a x-b e^{-c x}$, where $a \geq 0$ and $b, c>0$, then the corresponding standard measure of risk (66) is given with

$R(\tilde{x})=b E\left[e^{-c(\tilde{x}-E(\tilde{x}))}\right]$.

Note that the linear term of the utility function has no effect on the standard measure of risk.

Rescaling of the measure (71) according to (68) such that degenerate lotteries have zero risk leads to the risk measure

$R^{\prime}(\tilde{x})=b E\left[e^{-c(\tilde{x}-E(\tilde{x}))}-1\right]$.

The standard risk-value model (69) corresponding to the linear plus exponential utility model, finally, implies the risk measure

$R^{*}(\tilde{x})=b e^{-c E(\tilde{x})} E\left[e^{-c(\tilde{x}-E(\tilde{x}))}-1\right]$,

where, obviously, $\phi(x)=e^{-c x}$ is a monotonically decreasing positive real function. The measures (71), (72), and (73) of perceived risk all have the appealing properties of the standard measure of risk (66). They are zero for degenerate lotteries, they fit into the standard risk-value model (69), and adding a positive constant to all outcomes of a given lottery decreases its risk. It should be noted that these risk measures are closely related to Sarin's measure (41) and to Weber's measure (43).

\section{Empirical research on risk judgements}

There are quite a lot of empirical studies investigating judgements of perceived risk. For more comprehensive overviews see Keller [36], E.U. Weber and Bottom [65] and Weber [68]). Much of the empirical evidence stated in the sequel has been gained through studies investigating the reasonableness of one or more of the risk measures presented above or their respective axioms.

Overall these studies show that there is no model which is clearly superior to all the others in modelling risk judgements. However, there are some results which appear to be pretty stable across different empirical studies.

\section{- Subjects can give reliable risk judgements.}

A standard argument against measuring the riskiness of gambles is that subjects might not know what the riskiness of a gamble means to them. However, empirical studies have shown that subjects have a definite notion about the perceived riskiness of gambles and can give reliable risk judgements. Subjects were asked to rate alternatives according to their perceived riskiness twice. Ratings which were done two weeks later were remarkably identical to the original ratings. Even a cross cultural comparison yielded nearly identical results in those judgements. Keller, Sarin and Weber [37]) compared risk judgements for lotteries from students in California and Germany. The average judgements were 
practically identical. In addition, the degree to which these risk judgements followed certain basic properties, e.g., decreasing under positive translations, or disconfirmed some measures, e.g., Luce's logarithmic measure (28), was the same for both groups.

\section{- Risk is not just negative preference.}

A number of studies has shown that risk judgements cannot be easily explained as negative preference (see, e.g., Luce and E.U. Weber [44], Payne [49], and E.U. Weber and Botton [65]). E.U. Weber, Anderson, and Birnbaum [67] asked people to judge the riskiness and the attractiveness of lotteries. They found that both ratings were negatively correlated. However, the two tasks showed systematic differences in the rank order of judgements, thus indicating that perceived riskiness and preference are two distinct concepts.

\section{- Risk is not represented by variance.}

Consider the three two-outcome gambles $g_{1}=(\$ 10, .5, \$-$ $10), g_{2}=(\$ 20, .2, \$-5)$, and $g_{3}=(\$ 5, .8, \$-20)$, which all have equal mean and variance. Coombs and Bowen [22] as well as Keller, Sarin and Weber [37] found that subjects, asked about riskiness judgements, consistently judge $g_{3}$ or $g_{2}$ to be more risky than $g_{1}$. This finding shows that a classical risk measure such as the variance is, in general, not suitable to numerically represent a risk ordering.

\section{- Risk increases with an increase in variance or expected} loss.

To empirically test their distributive model (17), Coombs and Huang $[23,24]$ asked people to judge the riskiness of different two-outcome gambles $(y, p, z)$ with uniform probabilities, i.e. with $p=0.5$. Thereby they clearly found that perceived risk of a lottery increases when variance or expected loss of the lottery are increased. This finding implies that, in particular, perceived risk of a lottery increases when the range of the lottery is increased or when all outcomes of the lottery are multiplied by a constant with absolut value greater than one. Together, the last two results mean that an increase in variance is sufficient but by no means necessary for an increase in perceived risk.

\section{- Risk decreases under positive translations.}

Testing their distributive model (17), Coombs and Huang $[23,24]$ also found that perceived risk of a lottery decreases under positive translations, i.e., if a positive constant is added to all outcomes of a gamble. This result has been confirmed by the study of Keller, Sarin and Weber [37]. Appearently, it indicates that people's risk judgements are not location free. As mentioned in Sect. 6, this empirical finding has been the starting point for the derivation of Sarin's risk measure (41).

However, there is some doubt if these studies really elicited pure risk judgements. It cannot be excluded that people not strictly separated risk from preference judgements. For that reason, e.g., Weber [68] suggests to use Sarin's risk measure in the location free form (43). As mentioned in Sects. 8 and 9, also Fishburn as well as Jia and Dyer maintain the attitude that risk measures should be translation invariant.

\section{- Risk increases if a gamble is repeated several times.}

Repetition of gambles, up to now, has explicitly only been treated within Coombs psychophysics of risk (cf. Sect. 3). Using different two-outcome gambles $(y, p, z)$ with uniform probabilities, i.e. with $p=0.5$, Coombs and Meyer [26] have shown that the risk of a lottery increases when it is played several times. However, as mentioned in Sect. 5, many psychologists believe that the risk of a gamble that is repeated $\mathrm{n}$ times is less than $\mathrm{n}$ times the risk of the gamble played once.

\section{- The expectation principle may be violated.}

Keller, Sarin and Weber [37] elicited subjects' risk judgements where the gambles presented were those introduced by Allais [4] leading to the well-known Allais-paradoxa. They found that subjects violate the expectation principle. However, these violations occur less frequently than for preference judgements (E.U. Weber and Bottom [65]).

- Risk judgements additivly combine loss and gain components of gambles.

E.U. Weber and Bottom [65] have shown in two experiments that there is little support for Fishburn's multiplicative combination of risk measures depending on gains and losses. The experimental results can be explained by the CER-model of Luce and E.U. Weber [44].

\section{Risk measurement under partial probability information}

All of the risk measures discussed so far refer to the riskiness of alternatives that can be described by precise probability distributions over possible one-dimensional outcomes. In practice, however, situations of partial probability information are prevailing. Such situations of "partial ignorance" (Luce and Raiffa [43]) or "ambiguity" (Ellsberg [27]) are simply characterized by uncertainty about probabilities of outcomes.

A well-known example of a decision situation under partial probability information is Ellsberg's [27] so-called threecolour-problem. In this problem, an urn containing 90 balls is presented. 30 of these balls are known to be red. The remaining ones are known to be black or yellow, but with unknown proportion. From this urn, exactly one ball is to be drawn randomly. The alternatives are different bets on colors or pairs of colors, respectively. In this situation, obviously, the probability of red is $1 / 3$ and the probabilities of black or yellow are known to be between 0 and $2 / 3$ but uncertain. Camerer and Weber [17] point out the relevance of ambiguity for a wide range of professions and disciplines. They give a review of models that have been proposed to accomodate ambiguity.

There is some empirical evidence that in practical economic situations under partial probability information, e.g. project evaluation, decision makers use some sort of generalized mean-risk decision rule (cf. Brachinger and Schubert [15]). Modelling that kind of decision behavior presupposes a theory of risk under conditions of partial probability information. Based on the emprical evidence that already guided 
Fishburn's risk study (cf. Sect. 8), Brachinger [10, 14] developed a theory of risk under conditions of partial probability information in which gains and losses are treated separately. First, a theory of pure risk under partial probability information is developed where only potential losses are affected. Then, measures of speculative risk are proposed that allow for effects of potential gains on perceived risk.

Brachinger starts from the idea of a $m$-dimensional vector $X=\left(x_{1}, \ldots, x_{m}\right) \in \mathbb{R}^{m}$ of possible outcome values, $m \geq 2$. Partial probability information on $X$ is covered by any (non-empty) subset $\mathscr{P}$, with $|\mathscr{P}|>1$, of the set

$\mathscr{P}_{\mathscr{Z}}:=\left\{\mathbf{p}=\left(p_{1}, \ldots, p_{m}\right)^{\prime} \mid p_{i} \geq 0, \sum_{i=1}^{m} p_{i}=1\right\}$

of all $m$-dimensional discrete probability distributions, $p_{i}=$ $p\left(x_{i}\right)$. Thereby, $\mathscr{P}$ is to be interpreted as the set of all probability distributions compatible with the available information on the likelihood of the outcomes of $X$. In Ellsberg's three-colour-problem, e.g., partial probability information is covered by

$\mathscr{P}=\left\{\mathrm{p} \mid p_{1}=\frac{1}{3} \wedge p_{2}=\lambda \wedge p_{3}=\frac{2}{3}-\lambda ; \lambda \in\left[0, \frac{2}{3}\right]\right\}$

The most important practical cases of partial probability information are those where the decision maker is able to (not necessarily completely) rank the outcomes of a given vector according to their likelihood or where he or she disposes of interval-valued probabilities of these outcomes. It can be shown that in all of these cases the probability information $\mathscr{P}$ is linear in the sense that it allows a description

$$
\mathscr{P}=\left\{\mathbf{p} \in \mathscr{P}_{\mathbb{Z}} \mid \mathbf{B} \mathbf{p} \geq \mathbf{b}\right\} \quad,
$$

where $\mathbf{B}$ is $\mathbf{a}(k \times m)$-dimensional matrix and $\mathbf{b}$ is a $k$ dimensional vector.

According to the idea of partial probability information on a finite-dimensional vector of possible outcomes the theory of risk under partial probability information is, for any given finite dimension $m$, formulated in terms of the set of all pairs $(X, \mathscr{P})$, where $X \in \mathbb{R}^{m}$ is an $m$-dimensional vector of potential outcome values and $\mathscr{P} \subset \mathscr{P}_{\mathcal{Z}}$ a partial probability information on $X$. As Fishburn, Brachinger adopts the position that risk judgements are location free. Therefore, he presumes that some target value can be identified so that every value smaller than the target value is viewed as risky and is called a loss. Every value as large as the target value is viewed as non-risky and is called a gain. Without loss of generality, for convenience, this target value is set at zero. Furthermore, it is assumed that outcome preference increases in $x$.

Each vector $X=\left(x_{1}, \ldots, x_{m}\right) \in \mathbb{R}^{m}$ is decomposed into its corresponding loss vector given by

$$
\begin{aligned}
X^{+} & :=\left(x_{1}^{+}, \ldots, x_{m}^{+}\right) \\
& :=\left(\chi^{+}\left(-x_{1}\right) \cdot-x_{1}, \ldots, \chi^{+}\left(-x_{m}\right) \cdot-x_{m}\right),
\end{aligned}
$$

and its corresponding gain vector given by

$$
\begin{aligned}
X^{-} & :=\left(x_{1}^{-}, \ldots, x_{m}^{-}\right) \\
& :=\left(\chi^{+}\left(x_{1}\right) \cdot x_{1}, \ldots, \chi^{+}\left(x_{m}\right) \cdot x_{m}\right),
\end{aligned}
$$

where $\chi^{+}$is the characteristic function of the positive reals. For every vector $X \in \mathbb{R}^{m}$, its loss vector $X^{+}$and its gain vector $X^{-}$are $m$-dimensional vectors with non-negative components, $X^{+}, X^{-} \in \mathbb{R}_{0+}^{m}$. The loss vector summarizes all potential losses and the gain vector all potential gains of a given vector.

With regard to its pure risk every pair $(X, \mathscr{P})$ is characterized by the corresponding pair $\left(X^{+}, \mathscr{P}\right)$. I.e., objects of the theory of pure risk under partial probability information are all pairs $\left(X^{+}, \mathscr{P}\right)$, where $X^{+} \in \mathbb{R}_{0+}^{m}$ is an $m$-dimensional vector of potential losses and $\mathscr{P} \subset \mathscr{P}_{\mathbb{Z}}$ a partial probability information. Each pair $\left(X^{+}, \mathscr{P}\right) \in \mathbb{R}_{0+}^{m} \times \wp\left(\mathscr{P}_{\mathbb{Z}}\right)$ will be called risk vector.

The theory of pure risk under partial probability information is based on a binary risk relation $\succsim$, "is at least as risky as", defined on $\mathbb{R}_{0+}^{m} \times \wp\left(\mathscr{P}_{\mathscr{E}}\right)$. This relation is assumed to be a quasi-order, i.e. reflexive and transitive. In a first axiom it is required that a risk vector is riskless if and only if there are no potential losses. In two further axioms, the two components of risk vectors are reflected on separately. A potential loss is the greater the more the corresponding outcome value deviates from the target value. According to that it is assumed that of two risk vectors with identical probability components one is riskier than the other if the loss vector of the former dominates the loss vector of the latter.

According to conventional notion, risk increases as the chance of losses increases. In its intuitive sense, risk is the worst that can happen under given conditions. In the case of a partial probability information $\mathscr{P}$, given a specified loss vector $X^{+}$, the "worst that can happen" is that probability distribution $\mathbf{p} \in \mathscr{P}$ which maximizes loss expectation. This leads to the claim that of two risk vectors $\left(X^{+}, \mathscr{P}\right)$ and $\left(X^{+}, \mathscr{R}\right)$ with identical loss vectors $X^{+}$one is riskier than the other if

$\sup _{\mathrm{p} \in \mathscr{\mathscr { N }} \mathrm{i}} \sum_{i=1}^{m} x_{i}^{+} p_{i} \underset{(-)}{>} \sup _{\mathrm{p} \in \mathscr{\mathscr { V }} /} \sum_{i=1}^{m} x_{i}^{+} p_{i}$

Finally, in a further axiom it is supposed that rescaling of the outcome values lets the risk relation $\succsim$ invariant.

The risk relation $\succsim$ is not assumed to be complete and can, beyond the structure fixed by the above axioms, be quite arbitrary. The intersection of all risk relations $\succsim$ on $\mathbb{R}_{0+}^{m} \times \wp\left(\mathscr{P}_{\mathbb{Z}}\right)$ is the smallest relation fulfilling these axioms. Therefore, Brachinger presumes that a risk measure should be a nonnegative, real-valued mapping

$R: \mathbb{R}_{0+}^{m} \times \wp\left(\mathscr{P}_{\mathbb{Z}}\right) \longrightarrow \mathbb{R}_{0+}^{1}$

which numerically represents the intersection of all risk relations $\succsim$ on $\mathbb{R}_{0+}^{m} \times \wp\left(\mathscr{P}_{\mathbb{Z}}\right)$. According to the first axiom on the risk relation $\succsim$ it should be equal to zero if and only if a risk vector's loss component is identical to the null vector. Further, analogous to Luce's theory of risk, it is assumed that the risk $R\left(X_{\alpha}^{+}, \mathscr{P}\right)$ of a risk vector $\left(X_{\alpha}^{+}, \mathscr{P}\right)$ with rescaled loss component, $X_{\alpha}^{+}:=\alpha X^{+}$, is some function of the risk $R\left(X^{+}, \mathscr{P}\right)$ and $\alpha$. More specifically, corresponding to equation (23) it is required that, for all risk vectors $\left(X^{+}, \mathscr{P}\right)$ and all $\alpha>0$, there is a real function $S$ with $S(1)=1$ such that

$$
R\left(X_{\alpha}^{+}, \mathscr{P}\right)=S(\alpha) R\left(X^{+}, \mathscr{P}\right) .
$$

Thereby, $S$ should be increasing and continuous. 
Finally, according to the generally accepted conception that risk measures should have a kind of expectation property, it is assumed that, for all risk vectors $\left(X^{+}, \mathscr{P}\right)$, there is a real function $T$ with

$R\left(X^{+}, \mathscr{P}\right)=T\left(E_{\mathscr{P}}\left(X^{+}\right)\right)$

where, for any random variable $\tilde{x}$,

$E_{\mathscr{P}}(\tilde{x}):=\sup _{\mathrm{p} \in \mathscr{P}} \sum_{i} p_{i} x_{i}$.

From these assumptions Brachinger derives the risk measure

$R_{k_{1}}^{k_{2}}\left(X^{+}, \mathscr{P}\right)=k_{1} \Phi\left(X^{+}, \mathscr{P}\right)^{k_{2}}:=k_{1}\left(\sup _{\mathrm{p} \in \mathscr{P}} \sum_{i=1}^{m} x_{i}^{+} p_{i}\right)^{k_{2}}$

where $k_{1}, k_{2}$ are positive real constants. Equation (84) shows that measurement of pure risk under partial probability information by means of a measure $R_{k_{1}}^{k_{2}}$ is measurement on a log-interval scale.

In a second part of his study, as in Fishburn's "Foundations of Risk Measurement", Brachinger considers measurement of speculative risk where risk-reducing effects of gains on perceived risk are explicitly taken into account. Based on empirical results provided by E.U. Weber and Bottom [65] and similar as in Luce and E.U.Weber's risk measure (39), but contrary to Fishburn's multiplicativity assumption (56), it is started off from the idea that positive and negative components of a vector of outcome values, i.e. possible gains and losses are combined additively to arrive at judgements of its riskiness.

With regard to its speculative risk every pair $(X, \mathscr{P})$ is characterized by the corresponding triple $\left(X^{+}, X^{-}, \mathscr{P}\right)$. I.e., objects of the theory of speculative risk under partial probability information are all generalized risk vectors $\left(X^{+}, X^{-}, \mathscr{P}\right)$, where $X^{+}, X^{-} \in \mathbb{R}_{0+}^{m}$ are $m$-dimensional vectors of potential losses or gains, respectively, and $\mathscr{P} \subset$ $\mathscr{P}_{\mathcal{B}}$ is a partial probability information.

Analogous to the axioms for pure risk measurement, the axioms for speculative risk measurement under partial probability information are based on a binary risk relation $\succsim$ defined on $\mathbb{R}_{0+}^{m} \times \mathbb{R}_{0+}^{m} \times \wp\left(\mathscr{P}_{\mathbb{Z}}\right)$, which is presumed to be a quasi-order. In a first axiom a certain kind of continuity is assumed by requiring that there is, for any measure $R$ of pure risk, a real constant $\gamma>0$ with

$$
\begin{aligned}
\left(X^{+}, X^{-}, \mathscr{P}\right) \sim(\mathbf{0}, \mathbf{0}, \mathscr{P}) & \Longleftrightarrow \\
R\left(X^{-}, \mathscr{P}\right) & =\gamma R\left(X^{+}, \mathscr{P}\right) .
\end{aligned}
$$

By this constant the decision maker's risk attitudes are charcterized. Risk-lovers prefer $\gamma<1$, risk-averters $\gamma>1 ; \gamma=1$ implies risk-neutrality. Note that according to axiom (85) pure risk may be overcompensated by possible gains. Therefore, this condition favors speculative risk measures that are additively separable in gains and losses.

In further axioms it is assumed that, ceteris paribus, generalized risk should increase with increasing amount and probability of loss and decrease with increasing possible gains and gain probability. Finally, rescaling of the outcome values should leave the risk ordering $\succsim$ invariant.

A measure of speculative risk under partial probability information should be a mapping
$R^{*}: \mathbb{R}_{0+}^{m} \times \mathbb{R}_{0+}^{m} \times \wp(\mathscr{P}) \longrightarrow \mathbb{R}$

with $R^{*}=0$ for every vector of outcome values which is riskless in the sense of (85). It should be separable in the sense that there are measures $R_{k_{1}}^{k_{2}}$, and $R_{l_{1}}^{l_{2}}$ and a function $F: \mathbb{R}_{0+}^{2} \longrightarrow \mathbb{R}$ such that for every generalized risk vector $\left(X^{+}, X^{-}, \mathscr{P}\right)$ holds

$\left.R^{*}\left(X^{+}, X^{-}, \mathscr{P}\right)=F\left[R_{k_{1}}^{k_{2}}\left(X^{+}, \mathscr{P}\right), R_{l_{1}}^{l_{2}}\left(X^{-}, \mathscr{P}\right)\right]\right]$.

Thereby, $F$ should be strictly increasing in the first variable and strictly decreasing in the second one. It should be linear-homogenous and fulfill certain additional technical properties. The measures $R_{k_{1}}^{k_{2}}$ and $R_{l_{1}}^{l_{2}}$ quantify the pure risk and the chances connected with the outcome vector $X$.

These assumptions lead to the three-parametric class

$$
\begin{aligned}
R^{*}\left(X^{+}, X^{-}, \mathscr{P}\right) & =R_{\gamma, \ell, k}^{*}\left(X^{+}, X^{-}, \mathscr{P}\right) \\
& :=\ell\left(\gamma^{k} \Phi\left(X^{+}, \mathscr{P}\right)^{k}-\Phi\left(X^{-}, \mathscr{P}\right)^{k}\right),
\end{aligned}
$$

with $\gamma, \ell, k>0$ (cf. Brachinger [12] [14]). Within this class of generalized risk measures the parameter $\gamma$ covers the decision maker's risk attitude. The parameter $k$ can be interpreted as sensitivity parameter. In case of $k>1$ the decision maker is sensitive, and in case of $k \leq 1$ he is insensitive against the extents $\Phi\left(X^{ \pm}, \mathscr{P}\right)$ of a generalized risk vector's perceived risk or perceived chance, respectively. The parameter $\ell$ expresses the importance the decision maker assigns to generalized risks. In case of $\ell>1$ the decision maker "plays up", and in case of $\ell \leq 1$ he "plays down" every non-zero generalized risk.

\section{Final remarks}

A reading of the literature on concepts and measurements of risk shows that there is, by now, a variety of theoretical approaches. Among these approaches it is difficult to select anyone as superior by convincing a priori arguments. Empirical data will be needed. The need for more empirical investigations to evaluate alternative measures of risk is obvious.

It seems quite realistic that some definitions of risk may be more useful when the objective is to predict choices under uncertainty while others may be superior predictors of introspective judgements of perceived risk. It has to be emphazised, however, that the risk ordering, which is the subject matter of all the theories reviewed, need not be related to the preference ordering in any simple way.

We hold that the research on perceived risk should be better integrated with the descriptive as well as the prescriptive models of decisions under risk (see Sarin and Weber [54] for a review of risk-value models and Brachinger [13] for a new model). A better understanding of risk judgements could help us to develop more realistic risk-value models. In the areas of strategic planning (Bowman [9]), investment (Albrecht [1]), insurance (Albrecht [2]) and, especially, in finance risk-return considerations are standard. The capital asset pricing model (cf., e.g., Sharpe [56]) is based on a variance-expected value model, thus a special case of riskreturn model. For the capital asset pricing model one has already tried to develop alternative risk-value foundations (see 
Weber [68] for an overview and a new model). However, all these economic applications rest on a deeper understanding of risk judgements.

Acknowledgement. The authors acknowledge the careful reading of an unknown referee.

\section{References}

1. Albrecht P (1993) Normal and lognormal shortfall risk. In: 3rd AFIRColloquium, Rome 2:417-430

2. Albrecht P (1996a) Zur Konzeptionalisierung von Risiko und Chance mit Anwendungen in den Finanz- und Versicherungsmärkten. In: Hübner U, Helten E, Albrecht P (eds) Recht und Ökonomie der Versicherung. Verlag Versicherungswirtschaft, Karlsruhe, pp 1-22

3. Albrecht P (1996b) Dimensionen des versicherungstechnischen Risikos. In: Hesberg D, Nell M, Schott W (eds) Risiko - Versicherung Markt. Verlag Versicherungswirtschaft, Karlsruhe, pp 325-339

4. Allais M (1953) Le comportement de l'homme rationel devant le risque: Critique des postulats et axiomes de l'école americaine. Econometrica 21:503-546

5. Aschenbrenner KM (1978) Expected Risk and Single-peaked Preferences. Acta Psychologica 42:343-356

6. Bayerische Rück (Hrsg) (1993) Risiko ist ein Konstrukt. Knesebeck, München

7. Bernoulli D (1738) Specimen theoriae novae de mensura sortis. Commentarii academiae scientarum imperialis petropolitanae 5:175-192

8. Bernoulli D (1954) Exposition of a New Theory on the Measurement of Risk (Übersetzung von [7]). Econometrica 22:23-36

9. Bowman EH (1982) Risk Seeking by Troubled Firms. Sloan Management Review Summer Volume:33-42

10. Brachinger HW (1988) Entscheidungen bei Risiko: Kritik am Bernoulli-Paradigma und entscheidungstheoretische Fundierung des sensitivitätsanalytischen Ansatzes. Habilitationsschrift, Universität Tübingen, Tübingen, Germany

11. Brachinger HW (1991) Mean-Risk Decision Analysis under Partial Information. In: Chikán A (ed) Progress in Decision, Utility and Risk Theory. Kluwer Academic Publishers, Dordrecht Boston London, pp 193-201

12. Brachinger HW (1994) Theory of Risk under Partial Probability Information. Working Paper Nr. 241, Institute for Economic and Social Sciences, University of Fribourg, Fribourg, Switzerland

13. Brachinger HW (1995) Weak Lexicographic Risk-Value Models. In: Derigs U, Bachem A, Drexl A (eds) OR-Proceedings 1994. Springer, Berlin Heidelberg New York, pp 495-499

14. Brachinger HW (1997) Entscheidungen, Risiko und Ambiguität (überarbeitete und ergänzte Fassung von [10]). Springer, Berlin Heidelberg New York (to appear)

15. Brachinger HW, Schubert R (1985) The robust decision concept: An application to project evaluation. Management International Review 25:34-43

16. Burgemeister J, Weber M (1993) Risiko und Akzeptanz von Industrieansiedlungen. Zeitschrift für Betriebswirtschaft 63:147-169

17. Camerer C, Weber M (1992) Recent Developments in Modelling Preferences: Uncertainty and Ambiguity. Journal of Risk and Uncertainty $5: 325-370$

18. Colson G (1981) Toward a Bipolar Theory of Risk. European Journal of Operations Research 6:352-359

19. Colson G (1985) Theories of Risk and MCDM. In: Fandel G, Spronk J (eds) Multiple Criteria Decision Methods and Applications. Springer, Berlin Heidelberg New York, pp 171-196

20. Colson G, Zeleny M (1980) Uncertain Prospects Ranking and Portfolio Analysis under the Conditions of Partial Information. Oelgeschlager, Gunn \& Hain, Cambridge MA, USA

21. Coombs CH (1973) The Mathematical Psychology of Risk and a Theory of Risky Decision Making. Working Paper, University of Michigan, Ann Arbor, USA
22. Coombs CH, Bowen JN (1971) A Test of VE-Theories of Risk and the Effect of the Central Limit Theorem. Acta Psychologica 35:15-28

23. Coombs CH, Huang LC (1970a) Polynominal Psychophysics of Risk. Journal of Mathematical Psychology 7:317-338

24. Coombs CH, Huang LC (1970b) Tests of a Portfolio Theory of Risk Preference. Journal of Experimental Psychology 85:23-39

25. Coombs CH, Lehner PE (1984) Conjoint Design and Analysis of the Bilinear Model: An Application to the Judgement of Risk. Journal of Mathematical Psychology 28:1-42

26. Coombs CH, Meyer DE (1969) Risk Preference in Coin-toss Games. Journal of Mathematical Psychology 6:514-527

27. Ellsberg D (1961) Risk, Ambiguity, and the Savage Axioms. Quarterly Journal of Economics 75:643-669

28. Fishburn PC (1977) Mean-Risk Analysis with Risk Associated with Below-Target Returns. The American Economic Review 67:116-126

29. Fishburn PC (1982) Foundations of Risk Measurement. II. Effects of Gains on Risk. Journal of Mathematical Psychology 25:226-242

30. Fishburn PC (1984) Foundations of Risk Measurement. I. Risk as Probable Loss. Management Science 30:396-406

31. Fishburn PC, Kochenberger GA (1979) Concepts, Theory, and Techniques: Two-Piece von Neumann-Morgenstern Utility Functions. Decision Sciences 10:503-518

32. Hershey JC, Schoemaker PJH (1980) Risk-Taking and Problem Context in the Domain of Losses: An Expected Utility Analysis. Journal of Risk and Insurance 47:111-132

33. Hershey JC, Kunreuther HC, Schoemaker PJH (1982) Sources of Bias in Assessment Procedures for Utility Functions. Management Science 28:936-954

34. Jia J, Dyer JS (1995) A Standard Measure of Risk and Risk-Value Models. Working Paper No. 1, Risk-Value Study Series, Department of Management Science and Information Systems, The Graduate School of Business, University of Texas at Austin, Management Science (to appear)

35. Kahneman D, Tversky A (1979) Prospect Theory: An Analysis of Decision Under Risk. Econometrica 47:263-291

36. Keller LR (1986) Twenty Years of Experiments on the Riskiness of Gambles. Working Paper, University of Irvine, Irvine, USA

37. Keller LR, Sarin RK, Weber M (1986) Empirical Investigation of some Properties of the Perceived Riskiness of Gambles. Organizational Behavior and Human Decision Processes 38:114-130

38. Knight F (1921) Risk, Uncertainty, and Profit. Boston

39. Krantz DH, Luce RD, Suppes P, Tversky A (1971) Foundations of Measurement. Academic Press, New York London

40. Laughhunn, DJ, Payne JW, Crum R (1980) Managerial Risk Preference for Below-Target Returns. Management Science 26:1238-1249

41. Luce RD (1980) Several Possible Measures of Risk. Theory and Decision 12:217-228

42. Luce RD (1981) Correction to: Several Possible Measures of Risk. Theory and Decision 13:381

43. Luce RD, Raiffa H (1957) Games and Decisions. John Wiley, New York

44. Luce RD, Weber EU (1986) An Axiomatic Theory of Conjoint, Expected Risk. Journal of Mathematical Psychology 30:188-205

45. Machina M (1982) Expected Utility Analysis without the Independence Axiom. Econometrica 50:277-323

46. Machina M, Rothschild M (1987) Risk. In: The New Palgrave, A Dictionary of Economics. The Macmillan Press, London

47. Markowitz HM (1952) Portfolio Selection. The Journal of Finance 7:77-91

48. Markowitz HM (1959) Portfolio Selection: Efficient Diversification of Investment. Yale University Press, New Haven, USA

49. Payne JW (1975) Relation of Perceived Risk to Preferences among Gambles. Journal of Experimental Psychology: Human Perception and Performance 104:86-94

50. Payne JW, Laughhunn DJ, Crum R (1980) Translation of Gambles and Aspiration Level Effects in Risky Choice Behavior. Management Science 26:1039-1060

51. Payne JW, Laughhunn DJ, Crum R (1981) Further Tests of Aspiration Level Effects in Risky Choice Behavior. Management Science 27:953958 
52. Pollatsek A, Tversky A (1970) A Theory of Risk. Journal of Mathematical Psychology 7:540-553

53. Sarin RK (1987) Some Extensions of Luce's Measures of Risk. Theory and Decision 22:125-141

54. Sarin RK, Weber M (1993) Risk-Value Models. European Journal of Operations Research 70:135-149

55. Schneeweiss H (1967) Entscheidungskriterien bei Risiko. Springer, Berlin Heidelberg New York

56. Sharpe WF (1964) Capital Asset Prices: A Theory of Market Equilibrium and Conditions of Risk. Journal of Finance 19:425-442

57. Slovic P (1967) The Relative Influence of Probabilities and Payoffs upon Perceived Risk of a Gamble. Psychonomic Science 9:223-224

58. Slovic P (1987) Perception of Risk. Science 236:280-285

59. Stone BK (1970) Risk, Return, and Equilibrium. MIT Press, Cambridge London

60. Stone BK (1973) A General Class of Three-Parameter Risk Measures. Journal of Finance 28:675-685

61. Tobin J (1958) Liquidity Preference as a Behavior Toward Risk. Review of Economic Studies 25:65-86

62. Weber EU (1984a) Combine and Conquer: A Joint Application of Conjoint and Functional Approaches to the Problem of Risk Measurement. Journal of Experimental Psychology, Human Perception and Performance 10:179-194

63. Weber EU (1984b) Risk as an Independent Variable in Risky Choice. Doctoral Dissertation, Harvard University, Cambridge MA, USA
64. Weber EU (1988) A Descriptive Measure of Risk. Acta Psychologica 69:185-203

65. Weber EU, Bottom WP (1989) Axiomatic Measures of Perceived Risk: Some Tests and Extensions. Journal of Behavioral Decision Making 2:113-131

66. Weber EU, Milliman RA (1996) Perceived Risk Attitudes: Relating Risk Perception to Risky Choice. Management Science (to appear)

67. Weber EU, Anderson C, Birnbaum MH (1992) A Theory of Perceived Risk and Attractiveness. Organizational Behavior and Human Decision Processes 52:492-523

68. Weber M (1990) Risikoentscheidungskalküle in der Finanzierungstheorie. Poeschel, Stuttgart

69. Weber M, Camerer C (1987) Recent Developments in Modelling Preferences under Risk. OR Spektrum 9:129-151

70. Yates JF (1992) (ed) Risk-Taking Behavior. John Wiley, Chichester New York etc

This article was processed by the authors with the valuable assistance of Uwe Steinhauser using the $\mathrm{LAT}_{\mathrm{E}} \mathrm{X}$ style file pljour 2 from Springer-Verlag. 